\title{
VACUUM CONDITIONING AND BEAM LIFETIME OF THE SPRING-8 STORAGE RING
}

\author{
N. Kumagai, T. Noda, H. Ohkuma*, M. Oishi, H. Saeki, K. Soutome, Y. Suzuki, \\ M. Takao, H. Tanaka, Y. Taniuchi and K. Watanabe, JASRI/SPring-8, \\ 323-2, Mihara, Mikazuki-cho,Sayo-gun, Hyogo 679-5198, Japan
}

\section{Abstract}

The installation of the vacuum system of the SPring-8 storage ring was completed during 1996. Most of the vacuum chambers are made of aluminum alloy. The main pumping system is a mixed one consisting of NEG strips, DIP, SIP, and LNP. In addition to the standard treatments for ultrahigh vacuum components, dust-controlled procedure was adopted in the manufacturing and the installation processes.

Beam commissioning of the SPring- 8 storage ring started in March 1997. Since then an integrated beam dose of 190Ah has been achieved up to now, and correspondingly, the dynamic pressure rise per stored beam current $(\cdot \mathrm{P} / \mathrm{I})$ decreased by two orders of magnitude. In the multi-bunch mode a lifetime of 65 hours at $70 \mathrm{~mA}$ were achieved after two years' operation. Since the vertical emittance is extremely small, the beam lifetime is limited not only by dynamic pressure but also by Touschek effect even in the multi-bunch mode. The contributions of the gas scattering lifetime and Touschek lifetime are estimated in the case of the SPring- 8 storage ring.

\section{INTRODUCTION}

In the synchrotron light source, due to the large desorption induced by the synchrotron radiation (SR), The dynamic pressure in the electron storage ring vacuum system is very high in the early stage of commissioning, in spite of the fact that a static ultrahigh vacuum environment has been achieved. The interactions between the electron beam and the gas molecules desorbed from the chamber surface result in beam loss and emittance growth. Many criteria must be considered when designing the vacuum system of an electron storage ring. The design, fabrication, and testing of the vacuum chambers have been described previously [1]-[3].

The operation of the vacuum system shows good features of quick beam self-cleaning. In this paper, the conditioning of the SPring- 8 vacuum system and beam lifetime are described. The figure of merit for vacuum conditioning is the stored beam lifetime.

\footnotetext{
*Corresponding author; Email:ohkuma@spring8.or.jp
}

\section{VACUUM SYSTEM OF THE SPring-8 STORAGE RING}

The vacuum system of the SPring- 8 storage ring is made of 44 normal cells and 4 long straight cells, isolated by all metal rf-shielded gate valves. Each normal cell is composed of three straight section chambers (SSC's) with an absorber, two bending magnet chambers (BMC's), two crotch chambers (CR's) equipped with an crotchabsorber, two rf-shielded gate valves, one dummy chamber (IDD), four bellows assembled chambers (BEC's). A dummy chamber will be replaced by the vacuum system of the insertion device one by one.

The vacuum chamber materials are mainly aluminum alloys to benefit from their low outgassing rate, high thermal conductivity, low radio activity, and so on. The SSC and the BMC are made of A6063-T5 aluminum alloy by extrusion with the cooling channels on the both sides. The SSC consists of a beam chamber and a slotisolated ante-chamber in which a pair of non-evaporable getter (NEG) strips, allowing for distributed pumping are installed. The BMC includes a rectangular pump channel in which a distributed ion pump (DIP) is installed, a beam chamber and a slot-isolated ante-chamber in which a NEG strip is contained. The beam chambers of the SSC and BMC are extruded with the same cross section in an ellipse $70 \mathrm{~mm}$ in width and $40 \mathrm{~mm}$ in height.

To achieve a beam lifetime of approximately 24 hours, the vacuum chamber with its pumping system should be designed so as to maintain the dynamic pressure of the order of $10^{-7} \mathrm{~Pa}$ or less with a stored beam of $100 \mathrm{~mA}$. In our vacuum system, SR is almost intercepted by the crotch-absorbers and absorbers placed just downstream and upstream of bending magnets.

The main pumping system is based on NEG strips which are used in the SSC's and BMC's to evacuate the scattered molecules, and lumped NEG pump (LNP) for evacuating mainly SR-induced gases at the crotch and absorber location. In addition to the NEG strip, a DIP is installed in along side the beam chamber of the BMC. A sputter ion pump (SIP) which assists the LNP is also used. They offer the total pumping speed of about 10,000 1/s $\left(=10 \mathrm{~m}^{3} / \mathrm{s}\right)$ for one unit cell. 
The photon absorbers are made from Glid-Cop because of high allowable thermal stress of $60 \mathrm{~kg} / \mathrm{mm}^{2}$, compared to $10 \mathrm{~kg} / \mathrm{mm}^{2}$ of OFHC. The absorbers have the structure in which particles such as reflected photons, photoelectron and SR-induced outgases are efficiently trapped. SR-induced outgases are evacuated locally by the high capacity pumping system before the outgases have a chance to bounce into the beam chamber. The part of the photon beam power from a bending magnet of 10.5 $\mathrm{kW}$, about $6.6 \mathrm{~kW}\left(34 \mathrm{~kW} / \mathrm{cm}^{2}\right)$ is irradiated at the crotchabsorber and the remaining beam power deposited at the absorbers placed downstream of the crotch-absorber.

\section{VACUUM CONDITIONING AND BEAM LIFETIME}

All of the SSC's, BMC's and IDD's were $150{ }^{\circ} \mathrm{C}$ prebaked in advance of the chamber installation in the storage ring tunnel in order to remove chamber warps which were caused by the construction process and welding. The NEG pumps were activated at the last stage of pre-baking procedure. Residual pressure after the pre-baking was the order of $10^{-8} \mathrm{~Pa}$ or less. All of the installation works were accomplished in a clean room or clean booth. After the installation of the vacuum system in the storage ring tunnel, the $140{ }^{\circ} \mathrm{C}$ baking of the vacuum system and the NEG activation were done. The installation of the vacuum system of the SPring- 8 storage ring was completed in October 1996. Before the first beam injection on March 13, 1997, ultimate static vacuum pressure reached to about $9 \times 10^{-9} \mathrm{~Pa}$ at the absorber section and $5 \times 10^{-9} \mathrm{~Pa}$ at the SSC section. A thermal outgassing rate $(q)$ of the extruded aluminum alloy chamber was measured for the $R \& D$ of the vacuum chamber of the SPring- 8 storage ring [4]. As a result, $q$ was $6.7 \times 10^{-9} \mathrm{~Pa} \cdot \mathrm{m}^{3} \cdot \mathrm{m}^{-2} \cdot \mathrm{s}^{-1}$ after a baking at $140{ }^{\circ} \mathrm{C}$ for 40 hours. Because total inner surface area of the vacuum chamber per one unit cell is about $23 \mathrm{~m}^{3}$, thermal outgassing amounts $(Q)$ is $1.5 \times 10^{-7} \mathrm{~Pa} \cdot \mathrm{m}^{3} \cdot \mathrm{s}^{-1}$. As previously mentioned total pumping speed $(S)$ of one unit cell is about $10,000 \mathrm{l} / \mathrm{s}\left(=10 \mathrm{~m}^{3} / \mathrm{s}\right)$. Therefore, $1.5 \times 10^{-8} \mathrm{~Pa}$ of the pressure $(=Q / S)$ is obtained. This value is good agreement with the ultimate static vacuum pressure after the installation and the baking.

The first stored beam was obtained in March 25,1997. With the beam stored, the pressure increased abruptly due to the photon induced gas loads. The amount of gas loads is proportional to the beam current and the molecular desorption yield so that at the early stage of commissioning, the gas loads mainly depend on the molecular desorption yield. The normalized pressure rise $(\bullet P / I)$ were measured as high as $3 \times 10^{-2} \mathrm{~Pa} / \mathrm{A}$ initially and reduced to $2 \times 10^{-5} \mathrm{~Pa} / \mathrm{A}$ before the summer shutdown period of 1997 . After this period, the beam was stored as high as possible at overnight in order to clean up the photon irradiating surfaces by accumulating the total beam dose. As a result, the integrated beam dose reached to $11 \mathrm{Ah}$ and the beam lifetime has increased from about 3 hours to 15 hours with a beam current of $20 \mathrm{~mA}$ after the summer shutdown period of 1997.

In the summer shutdown period of 1997, all of the NEG strips and LNP were re-activated. The dynamic pressure was reduced by about one second at the SSC section, and then the beam lifetime increased. In the summer shutdown period of 1998, NEG activation was made again. A significant effect, however, could be observed in the dynamic pressure and in the beam lifetime.

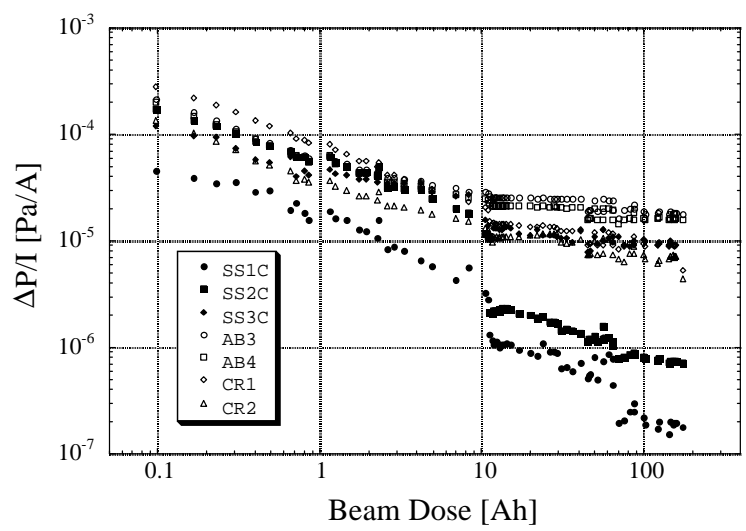

Figure 1: The normalized pressure rise $(\Delta P / I)$ as a function of the integrated beam dose.

The averaged pressure readings of the storage ring are $\leq 1 \times 10^{-8} \mathrm{~Pa}$ without electron beam, $1 \times 10^{-7} \mathrm{~Pa}$ at the SSC and $9 \times 10^{-7} \mathrm{~Pa}$ at the crotch and absorber location with a beam current of $70 \mathrm{~mA}$. After a continuous cleaning by the SR, the pressure rise due to the photon induced desorption (PID) effect was significantly reduced. Figure 1 shows the $\Delta P / I$ at the SSC, crotch and absorber section of No. 14 cell as a function of the integrated beam dose. The $\Delta P / I$ decreased by about two orders of magnitude after an accumulated beam dose of 190Ah. It shows the beam self-cleaning effect on the vacuum chamber. From this figure, in the early stage of commissioning $\Delta P / I$ decreases steadily with the slope of -0.57 . For the CR2,

$$
\Delta P / I=3.7 \times 10^{-5} D^{-0.57},
$$

where $D$ is the integrated beam dose. The photodesorption yields $(\eta)$ at the crotch-absorber section, CR2, is estimated using the following formula [5]:

$$
\begin{aligned}
& \eta=\Delta Q / \Delta N \\
& =K S_{\text {eff }}(\Delta P / I) /(\Delta N / I) \text { (molecules/photon), }
\end{aligned}
$$

where $\Delta Q\left(\mathrm{~Pa} \cdot \mathrm{m}^{3} \cdot \mathrm{s}^{-1}\right)=S_{\text {eff }} \Delta P$ is the gas desorption rate, $\mathrm{S}_{\text {eff }}$ $(\mathrm{Pa} / \mathrm{s}) \approx 1 \mathrm{~m}^{3}$ is the effective pumping speed of the LNP at the crotch-absorber section, $\Delta P$ is the pressure rise due to PID, $I(\mathrm{~A})$ is the beam current, $\Delta N($ photons $/ \mathrm{s}) \approx 8.05 \times 10^{20}$ $E I(\Delta \theta / 2 \bullet)$ is the photon flux irradiates on the crotchabsorber, $E=8 \mathrm{GeV}, \Delta \theta(\mathrm{rad}) \approx 0.0325 \mathrm{rad}$ is the photon span 
on the crotch-absorber, and $K \approx 2.26 \times 10^{20}$ (molecules $/ \mathrm{Pa} \cdot \mathrm{m}^{3}$ ) is the proportional constant. By using the data and formulas mentioned above, a photo-desorption yields at the crotch-absorber section is estimated,

$$
\eta_{\mathrm{CR} 2}=3.0 \times 10^{-4} D^{-0.57} \text { (molecules/photon) }
$$

At an accumulated beam dose of $10 \mathrm{Ah}, \eta_{\mathrm{CR} 2}=8.1 \times 10^{-5}$ molecules/photon was achieved. A photo-desorption yields at the range of the integrated beam dose larger than $10 \mathrm{Ah}$ may be smaller than this value. A correct value can not be estimated, because some of the ionization gauges malfunctioned due to the effect of the photoelectron induced by SR, especially at the crotch-absorber and absorber section.

Figure 2 shows the product of the electron-beam current $(I)$ and the beam lifetime $(\tau)$ versus the accumulated beam dose on the multi-bunch mode and several bunch mode operation. The one of the user operation mode of the SPring- 8 storage ring is the multi-bunch mode where two third of the 2436 available RF buckets are filled continuously with electron. An integrated beam dose of 190Ah and a beam lifetime of 65 hours at $70 \mathrm{~mA}$ of the beam current were achieved in the multi-bunch mode after two years' operation. The lifetime in the several bunches mode is limited by Touschek effect.

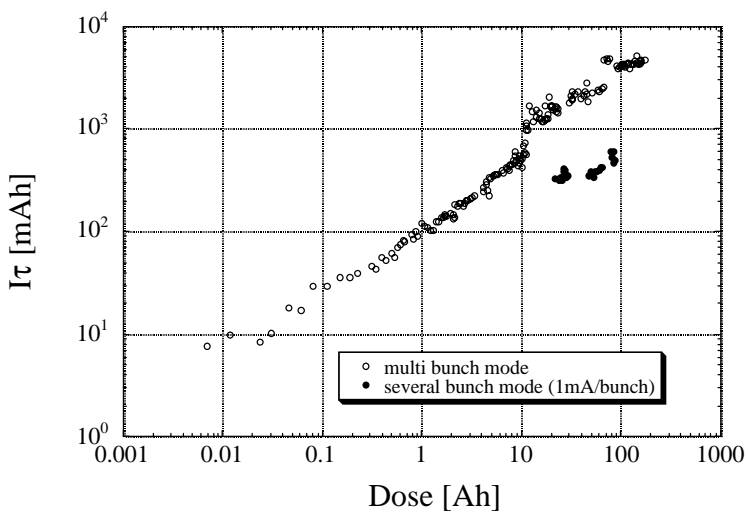

Figure 2: The product of the beam current $(I)$ and the beam lifetime $(\tau)$ versus the accumulated beam.

In multi-bunch mode of 2/3-filling, the beam current of $70 \mathrm{~mA}$ have been stored at user time, which means a population of $0.043 \mathrm{~mA} /$ bunch. In the SPring- 8 storage ring, the beam lifetime in the uniform filling mode is significantly longer than that in the partial-filling mode as shown in Fig. 3. In the electron storage ring, electron beam traps ions, which are produced by collisions of the electron beam with the residual gas molecules. Electrons and trapped ions, interact with each other and cause the instability. Ions are always trapped and electron beam size may be growth, so that the beam lifetime is longer in the uniform filling.

Another operation mode is several bunch mode. The 21-bunch mode (21 equally spaced bunches), the 21bunch train mode (21 equally spaced 3- or 7-bunches trains), 10-bunch + partially filled multi-bunch, and so on are used at the user time. 1 or $0.5 \mathrm{~mA} /$ bunch routinely stored at the several bunch mode of the user time. The vacuum pressure in the several bunch mode is as same as in the multi-bunch mode (2/3-filling).

Touschek lifetime is about 6 hours at the single bunch operation (1mA/bunch) of the nominal machine parameter of the SPring-8 storage ring [6]. As the total lifetime $(\tau)$ in the $2 / 3$-filling mode is 65 hours at the beam current of $70 \mathrm{~mA}$, gas scattering lifetime is estimated using a relationship: $1 / \tau=1 / \tau_{t}+1 / \tau_{\mathrm{g}}$, where $\tau_{\mathrm{t}}$ and $\tau_{\mathrm{g}}$ are Touschek and gas scattering lifetime, respectively. Since $\tau \approx 65$ hours and $\tau_{\mathrm{t}} \approx 120$ hours (at a population of $0.43 \mathrm{~mA} / \mathrm{bunch}$ ), $\tau_{\mathrm{g}} \approx 140$ hours.

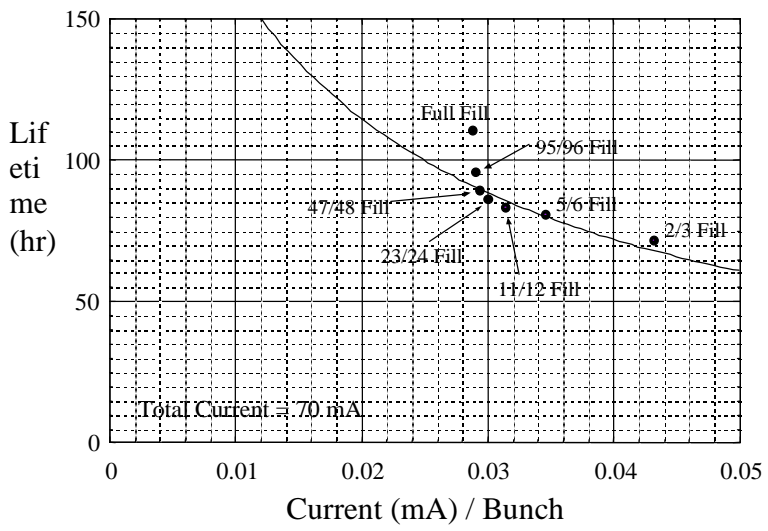

Figure 3: The beam lifetime versus the filling patterns of the multi-bunch mode.

\section{CONCLUSION}

The SPring-8 storage ring vacuum conditioning is progressing extremely well. A photo-desortion yields at the crotch-absorber section is $8.1 \times 10^{-5}$ molecules/photon or less. About 65 hours beam lifetime was achieved at a beam current of $70 \mathrm{~mA}$ in the multi-bunch mode. Touschek lifetime is about 6 hours at the single bunch operation, a population of $1 \mathrm{~mA} /$ bunch. A gas scattering lifetime is about 145 hours at a beam current of $70 \mathrm{~mA}$ with 2/3-filling mode operation.

\section{REFERENCES}

[1] H. Ohkuma, et al., Proc. of the 9th Meeting on Ultra High Vacuum Techniques for Accelerators and Storage Rings. KEK, March 3-4, 1994, KEK Proceedings 94-3, 29.

[2] K. Watanabe, et al., Proc. of the International Conference on Vacuum Science \& Technology, India, 1995, 58.

[3] H. Ohkuma, et al., Proc. of the 11th Symp. on Accelerator Sci. and Tech., Harima, Japan, 1997, 359.

[4] S. Yokouchi, et al., RIKEN Accel. Prog. Rep. 23 (1989) 134.

[5] G. Y. Hsiung, et al., J. Vac. Sci. Technol. A12 (1994)1639.

[6] M. Takao, et al., "Estimation of Betatoron Coupling and Vertical Dispersion for Spring-8 Storage Ring", presented in this proceedings. 